\title{
Assessment of geographical origin and production period of royal jelly by NMR metabolomics
}

Pierluigi Mazzei ${ }^{1,2^{*}} \mathbb{D}$, Alessandro Piccolo ${ }^{1,3^{*}}$, Marianna Brescia ${ }^{3}$ and Emilio Caprio ${ }^{3}$

\begin{abstract}
Background: Royal jelly (RJ) represents the most valuable product of beekeeping activities. The illegal introduction of RJ of uncertain quality/origin in European agrofood markets is not controlled, since the conventional analytical methods cannot unambiguously distinguish foreign from autochthonous RJ products. Here, we applied liquid-state NMR spectroscopy combined with chemometry to characterize the metabolic profile of representative Italian highquality RJ products and evaluate the discrimination capacity of this analytical technique.
\end{abstract}

Materials and methods: RJ samples were supplied by an Italian consortium of royal jellies producers and developed in the period May-July 2016. Samples were dissolved in a deuterated water solution brought at pH 7.3 and analysed through high resolution liquid-state NMR spectroscopy. 1D and 2D homo- and heteronuclear experiments were acquired to identify the RJ metabolomics profile. Proton NMR spectra were elaborated to conduct an NMR-based metabolomic approach based on PCA and DA chemometric analyses.

Results: The NMR-based metabolomics precisely and significantly ( $p$ value $<0.05$, according to Benjamini-Hochberg's test) authenticated the RJ products, by reliably tracing both their geographical and botanical origin, as well as their production period. In particular, Chinese RJ products exhibited a larger amount of citrate and lysine, accompanied by a lower content of 10-HDA. Our results showed that NMR spectroscopy can recognize low-quality fraudulent products and become an useful analytical tool to certify and trace the RJ molecular composition.

Conclusions: The application of NMR spectroscopy promises to enable the efficient protection of both the producers and consumers of high-quality royal jelly. In addition, the proposed method may be used in combination with the conventional melissopalynological methods for recognizing frauds.

Keywords: Liquid-state NMR, Royal jelly traceability, Multivariate statistical analysis, Territorial characteristics, Product authenticity

\section{Introduction}

Royal jelly (RJ) is biosynthesized, along with wax and venom, directly by honey bees as an hive product. It is secreted by the hypopharyngeal and mandibular glands of young bee workers aged between the 5th and the

\footnotetext{
*Correspondence: pmazzei@unisa.it; alessandro.piccolo@unina.it ${ }^{1}$ Centro Interdipartimentale sulla Risonanza Magnetica Nucleare per l'Ambiente, I'Agro-Alimentare ed i Nuovi Materiali (CERMANU), Università di Napoli Federico II, Via Università 100, 80055 Portici, Italy Full list of author information is available at the end of the article
}

14th day and serves to indiscriminately feed all larvae during first 3 days of their life. Only larvae selected to become queens continue to be fed with RJ throughout their life cycle [1]. The assumption of this functional food drives the phenotypic development of the bee female larva, allowing its transformation in a fertile bee queen instead of an unfertile bee worker. RJ consumption is further capable to significantly prolong the bee life cycle (5-6 years of the queen vs 1-6 months of the workers) [2]. 
RJ has a gelatinous consistency, often not homogenous due to the presence of undissolved granules of varying sizes. It appears as a whitish substance with differences in color tones depending on the type of pollen and honey with which the bees are fed at that time of RJ production. It has a distinctively sharp odor and taste, with an average density of $1.1 \mathrm{~g} \mathrm{~mL}^{-1}$. The main constituents of RJ are water, proteins, carbohydrates, lipids and mineral salts [3-5].

The RJ can be consumed by humans as food integrator, and it holds a high commercial value for its nutritional and nutraceutical properties. In fact, it is rich in various nutrients beneficial to humans, including proteins, sugars, vitamins, and a large number of bioactive substances, such as 10-hydroxy-2-decenoic acid (10HDA) [6]. Royal jelly is used as a health food and natural cosmetic in many countries [7]. It represents one of the most profitable hive products, since the price per gram is significantly greater than that of honey or pollen [1-7].

The ISO 12824 directive concerned with the quality of royal jelly was issued by BSI (British Standards Institution) only in 2016, although it is not yet made mandatory [8]. This directive specifies the production and sanitary requirements for RJ, establishes a series of analytical tests to control the RJ quality, and suggests methods to assess the botanical and geographical origin of royal jelly. In particular, the analysis of pollens is commonly recommended to reveal the vegetation, and, possibly, the geographical area from which the bees collect the nectars. However, this method is time-consuming, requires highly qualified personnel and exhibits several limitations. For example, it does not reveal the fraudulent practice by which RJ diagnostic original pollens are removed by ultrafiltration, and substituted with selected foreign pollens. Furthermore, the fact that no legislation yet imposes to RJ producers to indicate the origin of their product, favors the introduction in the EU market of low-quality non-European royal jellies, often mislabeled as Italian RJ. For example, in several cases it was found that Chinese RJ was fraudulently labelled as "made in Italy" and sold in Italy at an unjustified high price, at least 10-fold larger than the real commercial value. The competitive price of Chinese RJ products is a result of their low-quality, since their over-production is achieved using EU-forbidden antibiotics (i.e., chloramphenicol) and by neglecting the refrigeration requirements (cold chain logistic) during the long-range export [19]. Such conditions calls to develop alternative analytical techniques that are capable to identify the quality and, concomitantly, the geographical origin of royal jelly products.

Nuclear Magnetic Resonance (NMR) spectroscopy is a highly versatile physical-chemical technique that is progressively applied to evaluate the quality and trace the origin of a range of food matrices [9-11]. NMR techniques offer a number of advantages, including high reproducibility and possible direct analysis of products as such without preliminary sample extraction. This versatility allows the NMR spectroscopy to be applied to evaluate the quality and the origin of agroproducts, thereby becoming an useful tool against commercial frauds [1114]. Nevertheless, NMR spectroscopy applied to evaluate the molecular composition of bee-products has been upto-date limited to few works, such as on honey $[15,16]$, bee-wax [17], propolis [18], RJ [19], and RJ extracts [20, 21].

Therefore, the aim of this work was to develop a metabolomics approach, based on liquid-state NMR spectra of a number of royal jelly samples supplied and certified by the Italian Consortium of RJ products (COPAIT), with the purpose to evaluate their quality and authenticate their Italian origin. Moreover, we showed that the NMRbased metabolomics allowed not only to recognize Italian RJ products based on their different geographical origin, but also to discriminate them according to the production period and the type of blooms accessible to bees at the time of production.

\section{Materials and methods \\ Samples}

The Royal Jelly samples were provided by several Italian farms belonging to the COPAIT consortium, that is the most authoritative national association of Royal Jelly producers of Italy. To exclude any variation of RJ composition depending on farm-specific production, harvest, conservation and trade procedure, the samples were prepared by rigorously following the production and sanitary requirements dictated by the International Standard guide lines for Royal Jelly (BS ISO 12824:2016).

The RJ samples were produced between May and July 2016 by six Italian producers located in the Italian provinces of Bologna (Emilia Romagna region) and Grosseto (Toscana region). In Additional file 1: Table S1 we report the identification codes and the most relevant information on the involved farms, including geographical coordinates, average altitude and exact production date. Two farms were located in the province of Bologna (Bo1 and Bo6), while four were located in the province of Grosseto (Gr2, Gr3, Gr4 and Gr5). A supplementary COPAIT farm, situated in Andria (Puglia region) (An7), provided an additional sample (Additional file 1: Table S1).

The production/sampling days were established and scheduled by COPAIT farmers before the start of the production phase and ranged within the spring-summer period of the 2016. To identify any possible variation in 
RJ composition attributable to the production period, the $\mathrm{Gr} 3, \mathrm{Gr} 4$ and $\mathrm{Gr} 5$ farms provided samples produced in the same days (May 30, June 20, July 4 and 18, Additional file 1: Table S1).

With the aim to conduct a traceability study of RJ according to the pollen and the nectar collected by bees during the production period, we further considered several RJ samples which had been produced in monofloral conditions (beehives located in strict proximity of fields characterized by a unique or at least dominant botanical species) In detail, the condition of monofloral production occurred only in 1 day (June 13) of the whole production period, with a dominant pollen source of chestnut (c), trifolium ( $t$ ) or linden (l) for Bo1, Gr2 and Bo6, respectively (Additional file 1: Table S1). Such an evidence was assessed through the pollen traps method, as reported elsewhere (Additional file 1: Paragraph S1 and Fig. S1) [22-24].

Finally, based on the commercial menace represented by a massive import in Italy of RJ produced in extra-European countries (especially in China), we also examined two representative RJ samples produced in China (Ch1 and Ch2). The Italian COPAIT consortium provided these samples which resulted from a product sequestration done by the Italian Forestry Authority during an operation to contrast frauds (Chinese RJ introduced as false Italian RJ).

All samples were stored at $4{ }^{\circ} \mathrm{C}$ until NMR analysis. This storage procedure is critical, since temperatures higher than $8{ }^{\circ} \mathrm{C}$ may alter the RJ composition by the Maillard reaction between carbohydrates and amino acids, as revealed by the presence of fucosine $[25,26]$. However, the storage at the recommended temperature was not guaranteed for the Chinese RJ.

\section{NMR spectroscopy}

Ten (10) $\mathrm{mg}$ (fresh weight) of RJ were dissolved in $1 \mathrm{~mL}$ of a phosphate buffer $(0.2 \mathrm{M}, \mathrm{pH} 7.3)$ prepared with deuterated water $(99.8 \% \mathrm{D} 2 \mathrm{O} / \mathrm{H} 2 \mathrm{O}$, Armar Chemicals) and containing $0.05 \mathrm{mg} \mathrm{mL}^{-1}$ of 3-(Trimethylsilyl) propionic2,2,3,3-d4 acid (TMSPA, EurisoTop) as internal standard. This procedure led to a complete dissolution of royal jelly and no precipitation occurred within $72 \mathrm{~h}$. At least 6 replicates were prepared and examined for each RJ type.

NMR spectra were acquired at a temperature of $25 \pm 1{ }^{\circ} \mathrm{C}$ through a 9.4 Tesla Bruker Avance Magnet (Bruker Biospin, Rheinstetten, Germany) equipped with a BBI (Broad-Band Inverse) probe working at the resonance frequencies of 100 and $400 \mathrm{MHz}$ for ${ }^{13} \mathrm{C}$ and ${ }^{1} \mathrm{H}$ nuclei, respectively. For each single replicate it was acquired a proton spectrum combined with the onresonance presaturation (at an attenuation of $54 \pm 3 \mathrm{~dB}$ ) to suppress the residual water signal. The principal acquisition parameters for ${ }^{1} \mathrm{H}$ spectra consisted in 8 dummy scans, 128 scans, a $90^{\circ}$ pulse ranging within 8.8 and $9.9 \mu \mathrm{s}$ (at an attenuation of $-2 \mathrm{~dB}$ ), a spectral window of $16 \mathrm{ppm}(6410.256 \mathrm{~Hz}), 66560$ acquisition points and a recycle delay of $2 \mathrm{~s}$. The Fourier Transform was conducted by applying a two-fold zero-filling and an apodization of $0.2 \mathrm{~Hz}$.

The assignment of the most intense NMR signals detected in RJ metabolome was performed based on both previous literature [19] and interpretation of $2 \mathrm{D}$ NMR spectra of representative RJ samples. In particular, they following experiments were conducted: ${ }^{1} \mathrm{H}-{ }^{1} \mathrm{H}$ homonuclear ones such as COSY (COrrelation SpectroscopY), TOCSY (TOtal Correlation SpectroscopY) and NOESY (Nuclear Overhauser Effect SpectroscopY), and ${ }^{1} \mathrm{H}_{-}{ }^{13} \mathrm{C}$ heteronuclear ones such as HSQC (Heteronuclear Single-Quantum Coherence) and HMBC (Heteronuclear Multiple-Bond Coherence). The 2D NMR spectra were conducted by setting 16 dummy scans, 64 scans, and spectral windows of 16 and $300 \mathrm{ppm}$ for ${ }^{1} \mathrm{H}$ and ${ }^{13} \mathrm{C}$ nuclei, respectively. In all cases, 2D NMR spectra consisted in 2048 points (F2) for 256 experiments (F1). In particular, TOCSY and NOESY experiments were performed with 0.05 and $0.8 \mathrm{~s}$ of mixing times, respectively, whereas heteronuclear spectra were optimized according to a scalar and a "long-range" $J_{\mathrm{CH}}$ coupling of 145 and $8 \mathrm{~Hz}$, respectively.

For all 1D and 2D NMR spectra the frequency axes were calibrated by associating both TMSPA carbon and proton peaks at the chemical shift $\delta$ of $0 \mathrm{ppm}$. Spectral processing was conducted through the Bruker Topspin software (v. 4.0.2, Bruker Biospin, Rheinstetten, Germany).

\section{Multivariate statistical analyses}

${ }^{1} \mathrm{H}$ NMR spectra were equally divided into $n$-buckets of $0.01 \mathrm{ppm}(\approx 4 \mathrm{~Hz})$, thus generating 906 buckets per spectrum. The area resulting from the integration of each single bucket was divided by the area of TMSPA. The data matrix was Pareto-scaled to prevent the underestimation of relatively low intense NMR signals [27, 28].

Principal Component Analysis (PCA) was used to evaluate the data for identifying the variables (loadingvectors) discriminating different RJ types. The ANOVA (ANalysis Of VAriance) test was applied to assess the significance (Tukey Test at an $\alpha$ confidence level of 0.05 ) by which the most relevant variables (extrapolated through the evaluation of PCA loading-plots) contributed to differentiate the composition of RJ types [27-29]. In some cases, it was necessary to reach up to the PC5, though it often explained a relatively small amount of variance $(<20 \%)$. In these cases, we reported the results of ANOVA performed through the Benjamini-Hochberg's 
test for an $\alpha$ confidence level of 0.05 (Additional file 1: Tables S1 and S2).

The same data matrices used for PCA evaluation, but including only the variables that significantly discriminated among different RJ types, were also elaborated through the supervised Partial Least Square Discriminant analysis (PLS-DA) test [27]. PLS-DA permitted to assess the objective and significant ( $\alpha$ confidence level of 0.05 ) discrimination among the compositions of different RJ types by providing the percent of correct sample classification, correlation $\left(R^{2}\right)$ and prediction $\left(Q^{2}\right)$ fitness of the discriminant model, and by enabling the application of a cross-validation evaluation [27]. For this aim, we built a validation set, that was achieved by dividing all samples in either a training (discriminant model composed by samples which had been classified a priori) or a test (cross-validation model composed by unclassified samples) set. The repartition of samples into the two different subsets was conducted by accounting for the number of replicates per RJ type, and aiming to guarantee a number of replicates in the training set $>65 \%$. For example, 2, 4 or 8 were the number of replicates included into the test set for RJ types, that was composed of 6, 12 or 24 total replicates, respectively. This validation was repeated 5 times by randomly inverting, per each RJ type, the specific replicates included in the training and test set. The larger the percent of correct classification of test set samples, the better was the discrimination of the RJ samples. One representative score-plot (out of the five obtained) was shown for each PLS-DA cross-validation (see Additional file 1) [27]. ANOVA, PCA and PLS-DA tests were carried out by the XLStat software (v.2019, Addinsoft, Paris, France).

\section{Results and discussion}

\section{NMR-based metabolomic profiling of royal jelly}

A full ${ }^{1} \mathrm{H}$-NMR spectrum of a representative Italian RJ acquired by suppressing the solvent signal at $\approx 4.704 \mathrm{ppm}$ through the presaturation technique is shown in Additional file 1: Fig. S2. Generally, all proton NMR spectra of the RJ types revealed a relatively large number of signals $(>100)$ whose chemical shifts spread over the full proton spectral width (from 0.8 to $8.7 \mathrm{ppm}$ ), with intensities differing by several orders of magnitude. Such a heterogeneous spectral profile was expected, being the RJ composed by metabolites of different nature (i.e., amino acids, carbohydrates, fatty acids, etc.) and present in different concentrations. In all cases, the most intense proton signals were observed in hydroxy-alkyl (3.2-4.4 ppm) and alkyl (1.1-2.4 ppm) regions, whereas no signal was detected at frequencies greater than $8.7 \mathrm{ppm}$ (Additional file 1: Fig. S2).
The evaluation of NMR spectra of RJ samples and the assignment of related ${ }^{1} \mathrm{H}$ and ${ }^{13} \mathrm{C}$ NMR signals led to the identification of a typical metabolomic profile of an Italian Royal Jelly. Substantially, the signals assignment was conducted by: (i) interpreting mono- and bidimensional NMR spectra, (ii) comparing RJ NMR signals with those of known standards and NMR spectra libraries, and (iii) consulting pertinent previous literature $[15,16,19]$. In particular, the interpretation of 2D NMR spectra revealing through-coupling correlations, such as COSY (detecting geminal and vicinal ${ }^{1} \mathrm{H}-{ }^{1} \mathrm{H}$ correlations; Additional file 1: Fig. S3), HSQC and HMBC (detecting scalar ${ }^{1} \mathrm{H}_{-}{ }^{13} \mathrm{C}$ coupling and long-range ${ }^{1} \mathrm{H}-{ }^{13} \mathrm{C}$ connectivities, respectively; superimposition in Additional file 1: Fig. S4), resulted the most useful source of information.

Most of NMR signals detected in RJ metabolome were identified and the labels describing the molecular assignment are reported in both Figs. 1 ( ${ }^{1} \mathrm{H}$ NMR spectrum) and $2\left({ }^{1} \mathrm{H}_{-}{ }^{13} \mathrm{C}\right.$ HSQC $)$. In detail, Fig. 1 reports the four proton spectral regions (Figs. 1a-d) which include the most intense RJ signals (the full spectrum is shown in the top side of the figure and in Additional file 1: Fig. S2) and correspond to the ranges of $0.8-3.1 \mathrm{ppm}$ (Fig. 1a), 3.48-4.75 ppm (Fig. 1b), 5.18-5.95 ppm (Fig. 1c, $\times 3$ ) and 5.95-8.65 ppm (Fig. 1d, $\times 20$ ), respectively. The HSQC spectrum reports the assignment of the most intense signals of ${ }^{1} \mathrm{H}-{ }^{13} \mathrm{C}$ scalar correlation (Fig. 2). For the sake of clarity, colored ellipses were added to the figure to group the signals associated to the compounds specified in the figure legend.

The RJ metabolomic profiling indicated that the most intense signals detected in the hydroxy-alkyl region (Fig. 1b) are attributable to carbohydrates such as glucose, fructose and sucrose, whereas the pronounced peaks in the alkyl region (Fig. 1a) are ascribable to short-chain fatty acids. One of the most abundant compounds belonging to the latter molecular class is the 10-hydroxy-dec-2-enoic acid, namely $10-$ HDA. This compound is very peculiar, because it is naturally synthetized exclusively by the bees during the royal jelly secretion (therefore, also conventionally referred to as queen bee acid) and it as deemed responsible for the beneficial properties to human health. In fact, several works have shown the potential of 10-HDA in the pharmacological field by proving its role as antitumor, antibiotic, anti-inflammatory, immunomodulatory and neurogenic agent [29-33]. The evaluation of the spectral regions in Fig. 1a, b also indicated the presence, although to a lesser extent, of signals for amino acids (proline, lysine, arginine, valine and serine) and for several organic acids (citric acid, 3-hydroxybutyrric acid, pantothenic acid VB-5). Moreover, hydrogens bond to either $s p^{2}$-hybridized carbons (i.e., $\mathrm{H}$ bond to the $\mathrm{C} 2$ in the 10-HDA; Figs. 1 and 2) or anomeric carbons in saccharides were also evident in RJ spectra (Fig. 1c). Besides the abundance of carbohydrates, 
such as glucose, fructose and sucrose, this spectral region revealed the presence of maltose and ribose, whereas inositol became visible only through the HSQC experiment (Fig. 2). Finally, the signals detected in the spectral region shown in Fig. 1d are attributable to both aromatic amino acids, including tryptophan, phenylalanine and histidine, and nitrogen-based compounds ascribable to the principal building-blocks of nucleotides, such as nicotinamidic-(NIC), adenosinic-(ADE), guanosinic-(GUA) and uridinic-like (URI) structures. Unfortunately, we were not able to provide more detailed information on the structure of compounds belonging to the latter class, being the related NMR signals of very low intensity and, thus, scarcely informative. For example, although we have sufficient elements to reliably associate the signal at $8.3 \mathrm{ppm}$ to an adenosine-like structure, we cannot identify the specific adenosinic compound.

\section{Qualitative evaluation of different NMR-based metaboloma of Royal Jellies}

One of the main goals of this work was to employ the NMR-based metabolome of RJ samples to identify the origin and quality of royal jellies. This would represent an important outcome serving as a preliminary step to introduce a new and efficient analytical tool to guarantee to both producers and consumers the objective high quality of RJ products. Therefore, our experimental design consisted in evaluating the capability of the proposed approach to precisely and objectively discriminate among RJ samples (Additional file 1: Table S1), depending not only on the geographical origin (with a particular regard paid for the identification of Chinese RJ products), but also on important factors, such as the period of production and the botanical origin. However, by a qualitative comparison among ${ }^{1} \mathrm{H}$ NMR spectra, no specific biomarkers were found to be correlated to specific RJ types.

\section{Semi-quantitative assessment of different NMR-based metabolomes of Royal Jelly}

To semi-quantitatively evaluate whether the production conditions influenced the amount of specific RJ metabolites, the NMR results were elaborated by chemometric techniques. In particular, several data matrixes were created based on whole bucketed proton NMR spectra for each production conditions, and analyzed by multivariate statistical techniques, such as PCA and PLS-DA. This approach permits to take into account not only the existing variations between the potentially diagnostic signals of small intensity, but also to easily identify possible outliers.

\section{Geographical traceability of Royal Jelly}

The PCA score-plot based on ${ }^{1} \mathrm{H}$ NMR spectra of RJ samples produced by the Bologna and the Grosseto farms in the same day (May 30, 2016) and by the same procedure is reported in Fig. 3. The PC1 and PC5 explained $41.4 \%$ of the total variance and were the principal components that mostly represented the variables (metabolites) related to the RJ geographical origin. In fact, all Bo and Gr observations (Fig. 3) were neatly separated in the score plot as a function of their province of origin in Italy, and were mostly placed along the second and fourth quadrant thereby allowing to draw an hypothetical dotted line that divides the RJ types (Fig. 3). This result proves that the NMR-based metabolome may be potentially influenced from the different territorial characteristics of the RJ production site. The related loading-plot (Additional file 1: Fig. S5) revealed that the metabolites responsible for the significant (ANOVA results shown in Additional file 1: Table S2) discrimination of these RJ samples along the $\mathrm{PC} 1$ were citrate, valine and 3-hydroxybutyrate, while glucose, NIC, fructose, lysine and ADE were separated along the PC5. In particular, the RJ samples produced in Bologna exhibited a more abundant content of glucose, NIC, fructose, and lysine, but a lesser content of ADE, citrate, valine and 3-hydroxybutyrate than the RJ coming from Grosseto. The outcome of the application of PLSDA and related cross-validation technique (Additional file 1: Fig. S6) confirmed that it is possible to objectively discriminate the RJ produced in these different Italian provinces, with satisfying $\mathrm{Q}^{2}$ (goodness of prediction) and $R^{2}$ (goodness of fit) values for both PLS-DA axes [34].

Remarkably, the NMR-derived variables not only enabled the RJ discrimination based on the province of origin, but also as a function of the producing farms (Fig. 3), which are distant from each other only few tens of kilometers (Additional file 1: Table S1). In fact, RJ samples produced by different farms were placed in different areas of the score-plot, thereby revealing that the territory specific properties, such as microclimate, altitude, water and pollen quality/availability,

(See figure on next page.)

Fig. $1{ }^{1} \mathrm{H}$ NMR spectrum of a representative Royal Jelly sample. a-d The enlarged and magnified spectral regions within the chemical-shift ranges of 0.8-3.1, 3.15-4.75, 5.15-5.95 (×3 magnification) and 5.95-8.65 (×20 magnification) ppm, respectively. The figure reports the assignment of the most intense signals. The labels 10-HDA, URI, NIC, GUA and ADE refer to 10-hydroxy-2-decenoic acid and uridinic-, nicotinamidic-, guanosinic- and adenosinic-like structures, respectively 

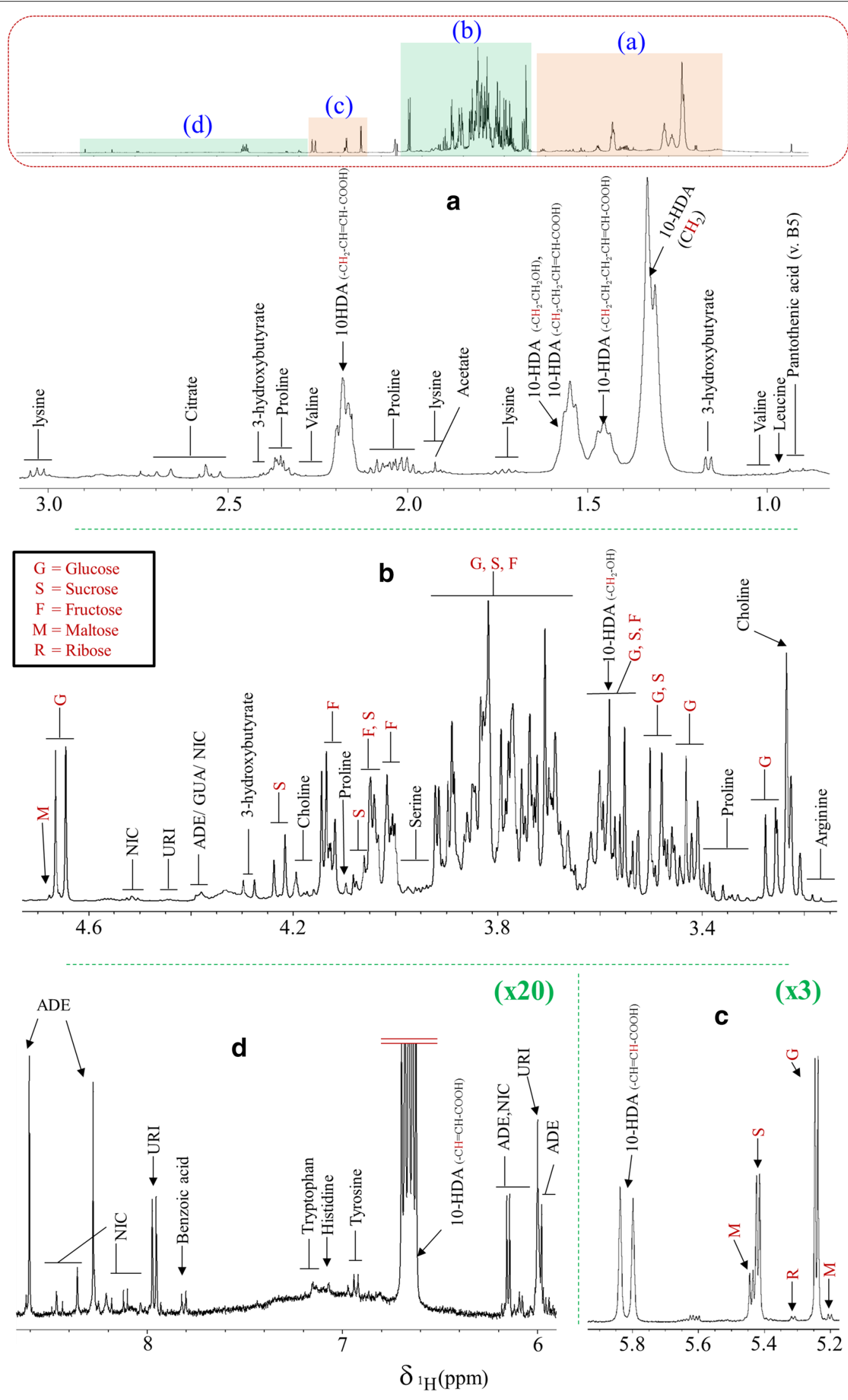

(x3)

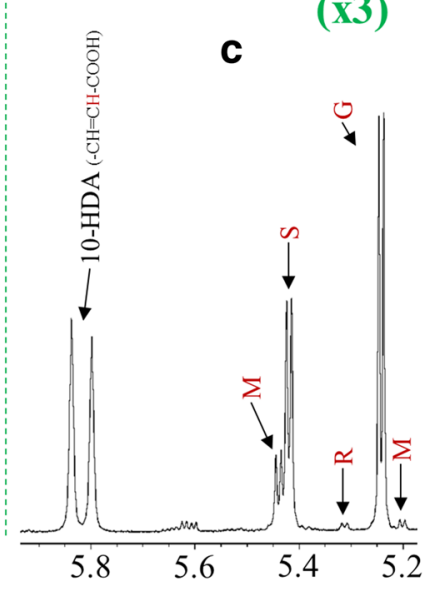




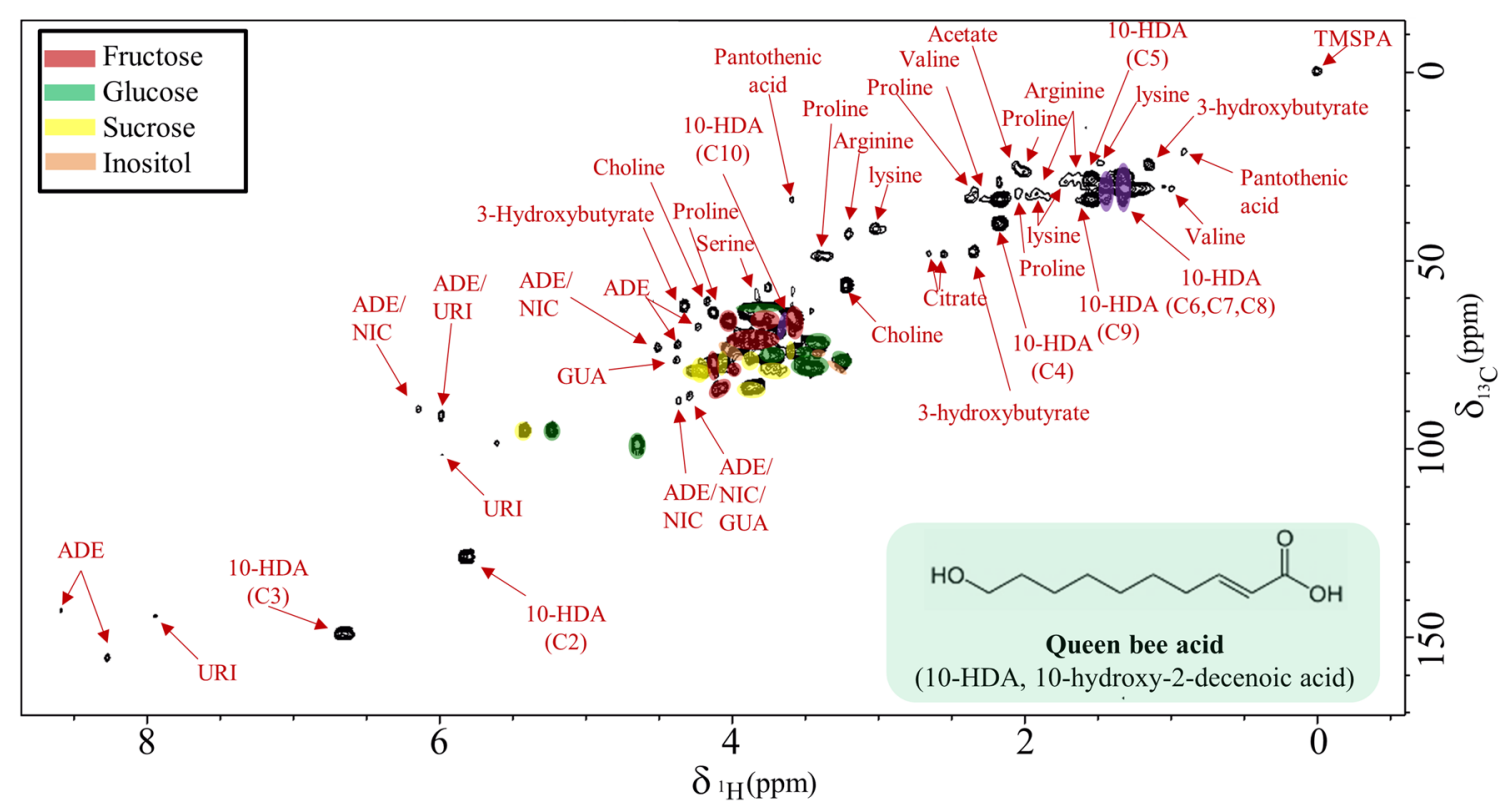

Fig. $22 \mathrm{D}^{1} \mathrm{H}-{ }^{13} \mathrm{C} \mathrm{HSQC} \mathrm{NMR} \mathrm{spectrum} \mathrm{of} \mathrm{a} \mathrm{representative} \mathrm{Royal} \mathrm{Jelly} \mathrm{sample.} \mathrm{The} \mathrm{figure} \mathrm{reports} \mathrm{the} \mathrm{assignment} \mathrm{of} \mathrm{the} \mathrm{most} \mathrm{intense} \mathrm{signals.} \mathrm{The}$ labels 10-HDA, URI, NIC, GUA and ADE refer to 10-hydroxy-2-decenoic acid and uridinic-, nicotinamidic-, guanosinic- and adenosinic-like structures, respectively. The green ellipse in the bottom-right side includes the chemical structure of 10-HDA compound

may influence the RJ biosynthesis by the bees. Thus, it is reasonable to infer that the larger the differences among the characteristics of the production sites, the greater is the diversity in NMR-based metabolomes of RJ products. The fact that Bo1 and the two Gr3, and Gr4 scores appeared, respectively, as the most negative and positive values in the PC1, was indicative of a composition with a respective small and large content of citrate, valine and 3-hydroxybutyrate. While RJ scores for Bo6, Gr2 and Gr5 could not be discriminated along the PC1 because of similar amounts of citrate, valine and 3-hydroxybutyrate, the variables associated to PC5 (glucose, NIC, fructose, lysine and ADE) allowed to significantly separate RJ types, as verified through the ANOVA Benjamini-Hochberg test (Additional file 1: Table S2). In particular, the RJ from Bo6 showed the greatest content of glucose, NIC, fructose and lysine, and the smallest amount of ADE.

The results of PLS-DA and cross-validation procedures applied to discriminate RJ samples produced in Bologna and Grosseto farms are shown in Additional file 1: Figs. S7 and S8. For both production sites, the $100 \%$ of unknown samples were correctly classified during the cross-validations. These findings indicate the validity of the adopted method in recognizing different RJ products on the basis of their molecular composition as revealed by NMR spectra, thereby underlining the potential of the NMR technique to precisely and objectively trace the origin of RJ products.

\section{Authentication of RJ products}

To verify the capacity of NMR spectroscopy to discriminate fraudulent RJ products, we compared the NMR-based metabolome of two Chinese RJ products, sequestered by Italian authorities as commercial frauds, with that of Italian RJ samples produced in the same day but in different farms of the provinces of Bologna, Grosseto and Andria. The PCA score-plot of Chinese RJ products (Fig. 4) were neatly separated from the Italian ones along the horizontal PC3, due to their significantly smaller amount of 10-HDA, that is commonly thought to be beneficial to human health [29-33]. Conversely, the Chinese RJ products were found rich in citrate and lysine (Additional file 1: Fig. S9; Tables S1). The poor content of 10-HDA found in Chinese RJ samples is in line with the results of Messia et al. [19] and with those showing the large content of such a queen bee acid in Italian RJ products [35]. It is to be noted that the difference between the Chinese and the Italian RJ samples was confirmed by PLS-DA and related cross-validation technique that showed a $\mathrm{Q}^{2}$ prediction goodness of 0.501 (Additional file 1: Fig. S10). Although an NMRbased method to identify Chinese RJ samples should be validated with a larger number of both replicates 


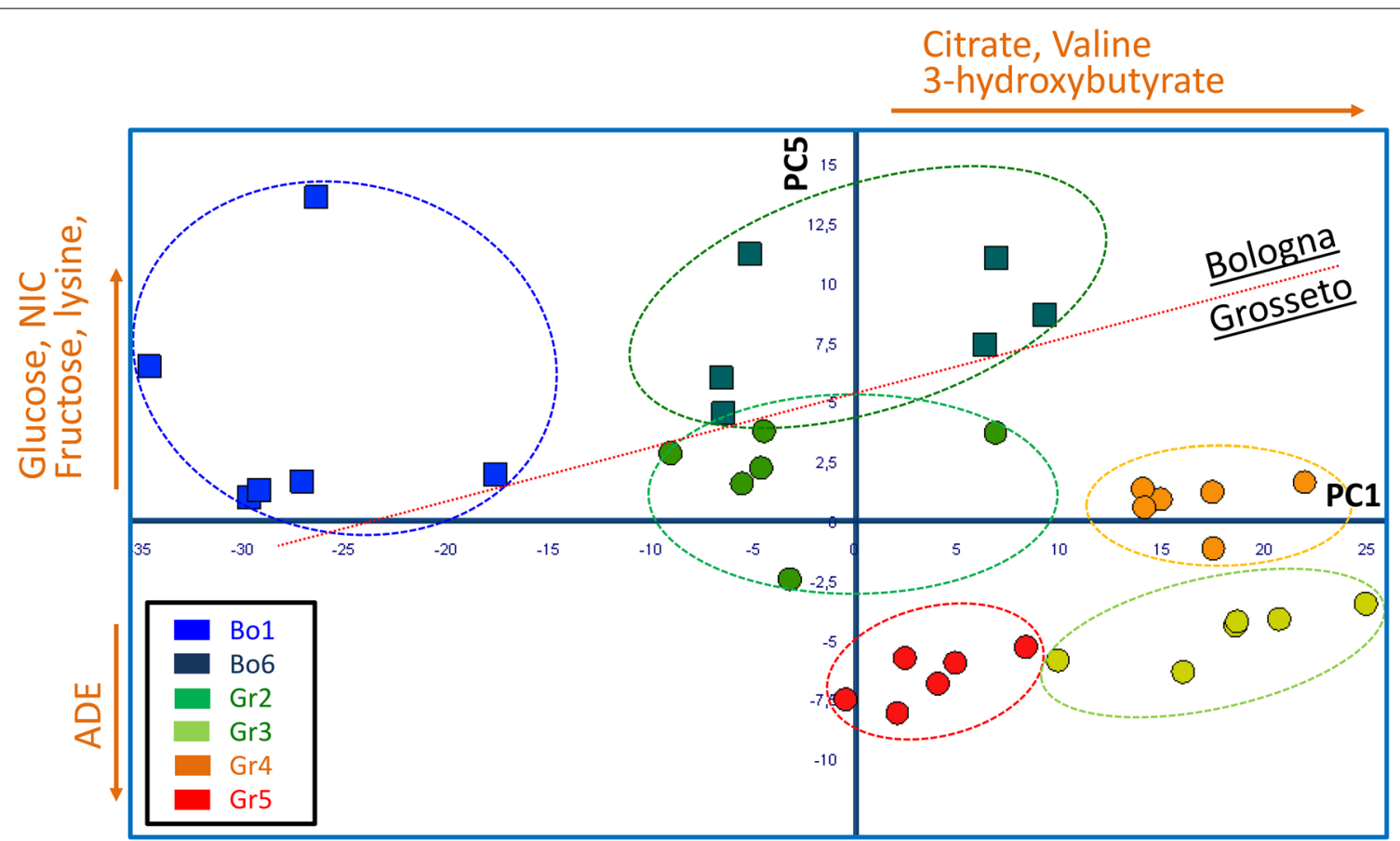

Fig. 3 PCA score-plot (41.4\% of total variance explained) based on ${ }^{1} \mathrm{H}$ NMR spectra of Royal Jelly samples produced by two Bologna (Bo1 and Bo6, squares) and four Grosseto (Gr2, Gr3, Gr4 and Gr5, circles) farms on the 30th of May 2016. The ellipses and the red dotted line were generated arbitrarily to indicatively group the RJ types and introduce a separation axis between the Bologna and Grosseto RJ samples, respectively. The name and orthogonal direction of most significant loading vectors involved in the differentiation among RJ types are reported along the plot borders (more details are given in Additional file 1: Fig. S5)

and types of RJ products from China, our preliminary results appear very promising.

Moreover, the variables associated to the vertical PC5 permitted a significant differentiation (Additional file 1: Tables S1) of Italian RJ products on the basis of the various province of origin. In particular, the RJ samples from Grosseto showed the largest amount of fructose and sucrose together with the smallest amount of ADE, whereas the opposite was true for the samples from Andria (Fig. 4, Additional file 1: Fig. S9).

\section{Traceability of RJ as a function of the production period}

The production period for royal jellies in Italy generally ranges from April to July and undergoes a significant variation in terms of influencing parameters. In fact, temperature fluctuations and water availability are expected to affect the physiology of bees involved in RJ biosynthesis, with indirect effects on RJ composition. To verify this aspect, we compared the ${ }^{1} \mathrm{H}$ NMR spectra of Royal Jelly samples produced by three Grosseto farms, Gr3, Gr4 and $\mathrm{Gr} 5$, in 4 days chosen within the RJ production period (May 30, June 20, July 4 and 18, and referred to as I, II, III and IV period, respectively).

We found that these RJ products were neatly separated in the score-plot built on the NMR-based metabolomes
(Fig. 5), thus suggesting a difference in the RJ composition due to the production period. In the case of farm Gr3 (Fig. 5a), the RJ types were separated along the horizontal PC2 because of the content of citrate, 3-hydroxybutyrate, proline and sucrose, whereas a further separation occurred along the vertical PC3 due to the amount of glucose, fructose and lysine (Fig. 5a, Additional file 1: Fig. S11A; Table S3). As for farm Gr4 (Fig. 5b), the RJ types were separated along the horizontal PC3 due to different amounts of glucose and fructose, while the differentiation along the vertical PC4 was dictated by different concentrations of fructose, sucrose, ADE and proline (Fig. 5b, Additional file 1: Fig. S11B; Table S3). Also for the farm Gr5 (Fig. 5c), all RJ produced at different times were well separated from each other. In particular, the samples produced in the II and IV period were placed in the most positive values of $\mathrm{PC} 1$ because of the largest abundance of proline, citrate, valine and sucrose, and of the least content of glucose and fructose (Fig. 5c, Additional file 1: Fig. S11C; Table S3). Conversely, the samples collected July 18 were separated from those produced in all other periods along the vertical $\mathrm{PC} 4$ axis, since they contained the larger amount of valine, 3-hydroxybutyrate, NIC and $\mathrm{ADE}$, and a significantly lower amount of sucrose (Fig. 5c, Additional file 1: Fig. S11C; Table S3). 


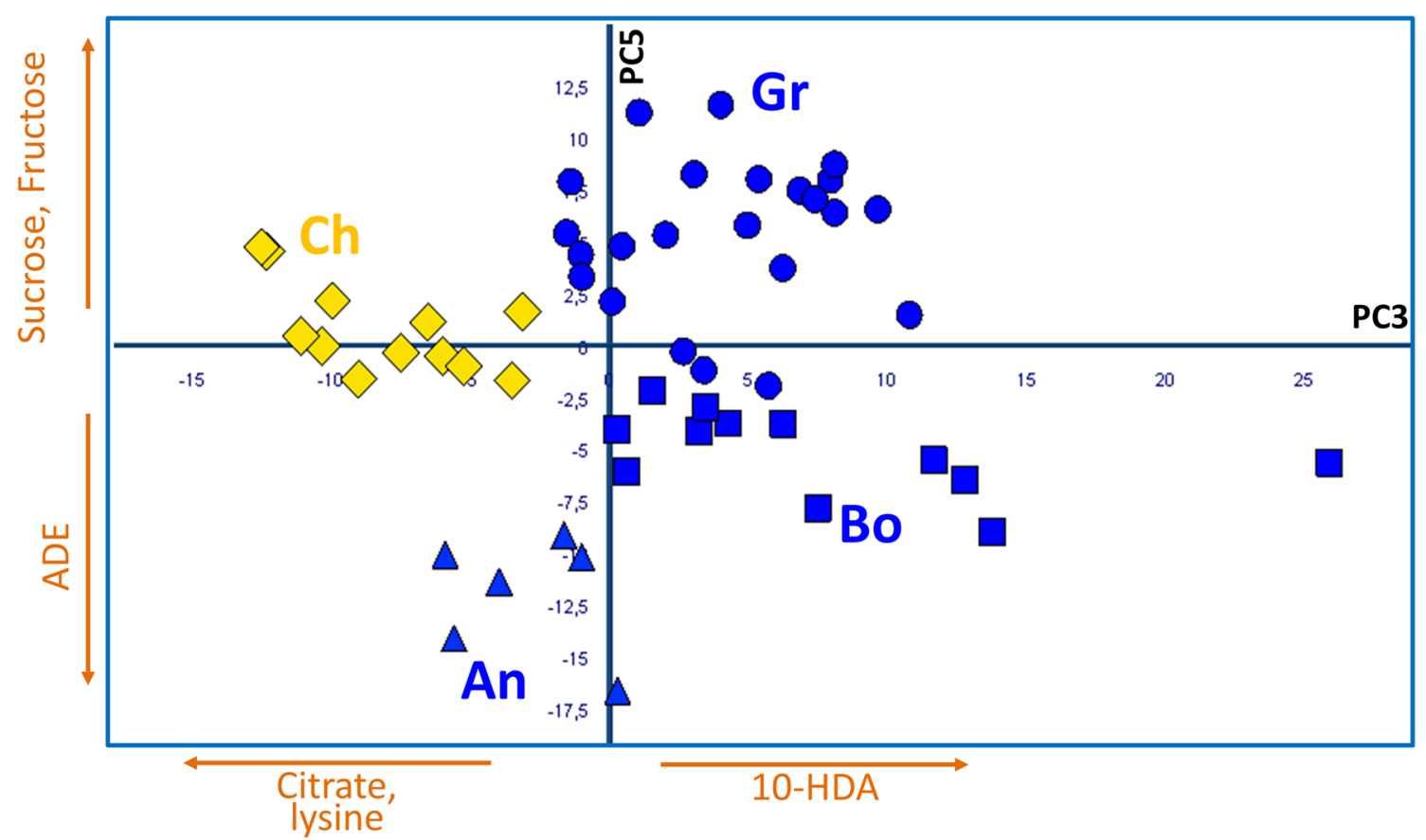

Fig. 4 PCA score-plot (13.7\% of total variance explained) based on ${ }^{1} H$ NMR spectra of Royal Jelly samples of Italian (Bologna, Grosseto and Andria samples are represented by blue squares, circles and triangles, respectively; produced the $8^{\text {th }}$ of July 2016) and Chinese (yellow rhombi; distributed in Italy in 2016) geographical origin. The name and orthogonal direction of most significant loading vectors involved in the differentiation among RJ types are reported along the plot borders (more details are reported in Supporting Fig. S9)

Our findings generally revealed that the RJ samples, even when produced in the same farm, showed a metabolome that varied with the production period, and depended on changes of metabolites such as carbohydrates, amino acids, nucleic acid derivatives, and organic acids (Fig. 5). An explanation may reside on the fact that RJ composition was presumably affected not only by microclimate factors but also by specific territorial conditions that varied from farm to farm. This suggests that a full exploitation of the NMR technique to authenticate RJ samples should rely on the establishment for each farm of several spectral databases, that also account for the variability due to the production period.

Nevertheless, we observed that for all the Gr3, Gr4, and Gr5 farms, RJ samples produced in the III period (July 4) fell in an isolated area of the respective score plots (Fig. $5 \mathrm{a}-\mathrm{c}$ ) because of a larger content of glucose and fructose. By consulting the archives of the Regional Climate Centre (https://www.meteotoscana.it/), we found that the week of July 4 had been very warm in the Grosseto area (maximum temperature $35^{\circ} \mathrm{C}$ at $200 \mathrm{~m}$ above the sea level), and that the III period was the warmest of all considered periods for the production of RJ samples. We can generally infer that a warm temperature correlates with the greatest concentration of fructose and glucose in RJ products.

\section{Traceability of RJ according to the botanical origin of pollens}

The identification of the botanical origin of pollens is an additional method to assess the quality and the geographical origin of RJ products. For example, Dimou et al. [22] showed that the analysis of pollen residues found in the royal jelly allows to recognize the geographical origin of the RJ product. However, it has been also shown that the melissopalynological analysis alone cannot represent an exhaustive evidence to ascertain the geographical origin and authentication of RJ products, since they can also be adulterated by fraudulently removing or adding granules of pollen [36]. Thus, it can expected that a synergy between melissopalynological analysis and ${ }^{1} \mathrm{H}$ NMR-based metabolomics may become a more reliable and objective approach to contrast the introduction of fraudulent RJ products.

On this basis, we compared the NMR-based PCA of RJ samples produced in the same day (June 13, 2016) by three Italian farms (Bo1, Bo6 and Gr5) and derived from a monofloral production related to flowers of either chestnut, trifolium or linden. The PCA score-plot of these RJ samples composed by $\mathrm{PC} 1$ and $\mathrm{PC} 2$ explained $58,2 \%$ of total variance of the NMR-based variables (Fig. 6). In line with previous works relating ${ }^{1} \mathrm{H}$ NMR spectra to the botanical origin of honeys $[15,16]$ we found that also the 
a

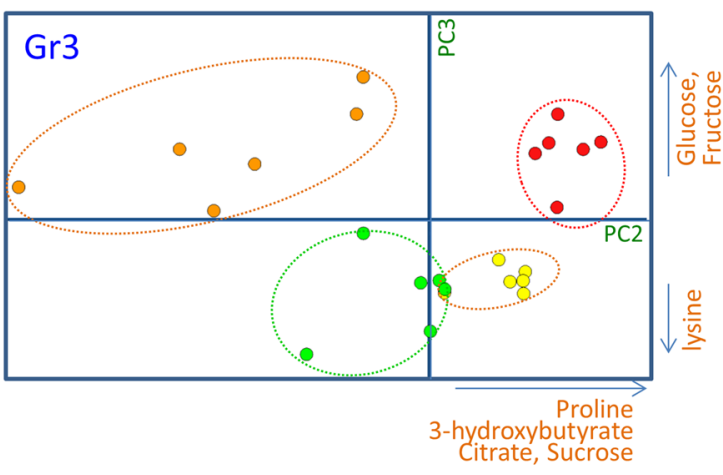

C

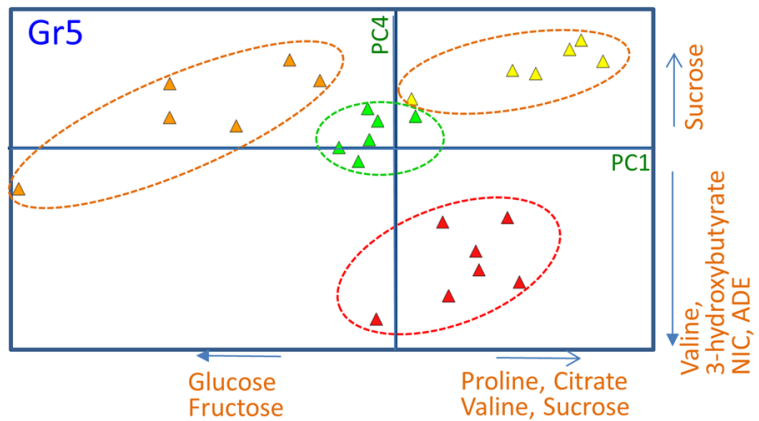

b

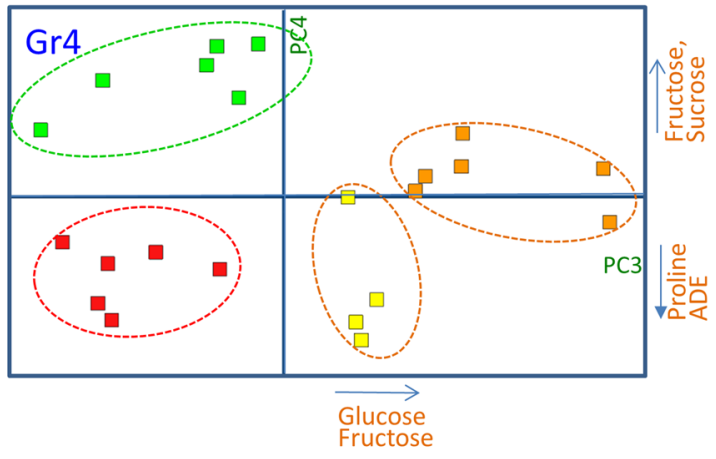

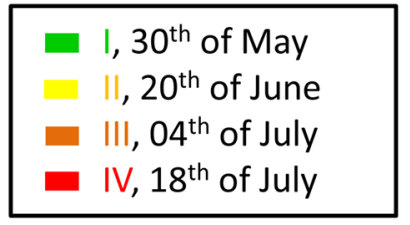

Fig. 5 PCA score-plots based on ${ }^{1} \mathrm{H}$ NMR spectra of Royal Jelly samples produced by three Grosseto farms (Gr3, circles, a; Gr4, squares, b; Gr5, triangles, $\mathbf{c}$ in different days (see legend). Total variance explained by the score-plots shown in Figs A, B and C corresponds to 37.5, 18.7 and $40.1 \%$, respectively. The ellipses were arbitrarily generated to indicatively group the different RJ types. The name and orthogonal direction of most significant loading vectors involved in the differentiation among RJ types are reported along the plot borders (more details are reported in Supporting Fig. S11)

RJ products were neatly discriminated according to the botanical origin (Fig. 6). In particular, the loading plot (Additional file 1: Fig. S12) revealed that the RJ derived from the chestnut pollen revealed a relatively small abundance of proline, valine, 10-HDA, 3-hydroxybutyrate and citrate (Fig. 6). While the content of these variables was almost similar in monofloral RJ samples of trifolium and linden, these latter types were separated from each other along the PC2 (Fig. 6) because of a larger abundance of aromatic amino acids, such as tryptophan, tyrosine and histidine, and a lesser amount of 10-HDA in the RJ sample produced with the trifolium pollen (Additional file 1: Fig. S12, Table S3). Therefore, these results indicate that the type and quantity of pollen available to bees determine the characteristics of the RJ metabolome, thus proving the NMR objective diagnostic power in evaluating the monofloral origin of RJ products. Unfortunately it was not possible to examine monofloral samples produced in the same site. Therefore, in line with our outcomes concerning the geographical traceability of RJs, we cannot exclude that a partial contribution in the metabolome differentiation was due to the different geographical origin of the three RJ types.

\section{Conclusions}

In this work we showed that NMR spectroscopy is a suitable and reliable analytical technique to evaluate the molecular characteristics of royal jelly, one of the most valuable bee-products. The typical primary metabolome of royal jelly was identified by assigning the most intense ${ }^{1} \mathrm{H}$ and ${ }^{13} \mathrm{C}$ signals detected in $1 \mathrm{D}$ and 2D NMR spectra. Moreover, the elaboration of NMR spectral results by powerful multivariate statistical methods, such as Principal Component Analysis and Partial Least Square Discriminant Analysis, allowed to precisely and objectively obtain the molecular fingerprint of specific royal jellies. Our results indicate that NMR spectroscopy represents a valid tool not only to authenticate royal jelly as such, but also to identify the specific molecular components that are directly related to the quality, geographical and botanical origin, and production period of royal jelly products. Furthermore, the combination of NMR spectroscopy with multivariate analysis applied here allowed 


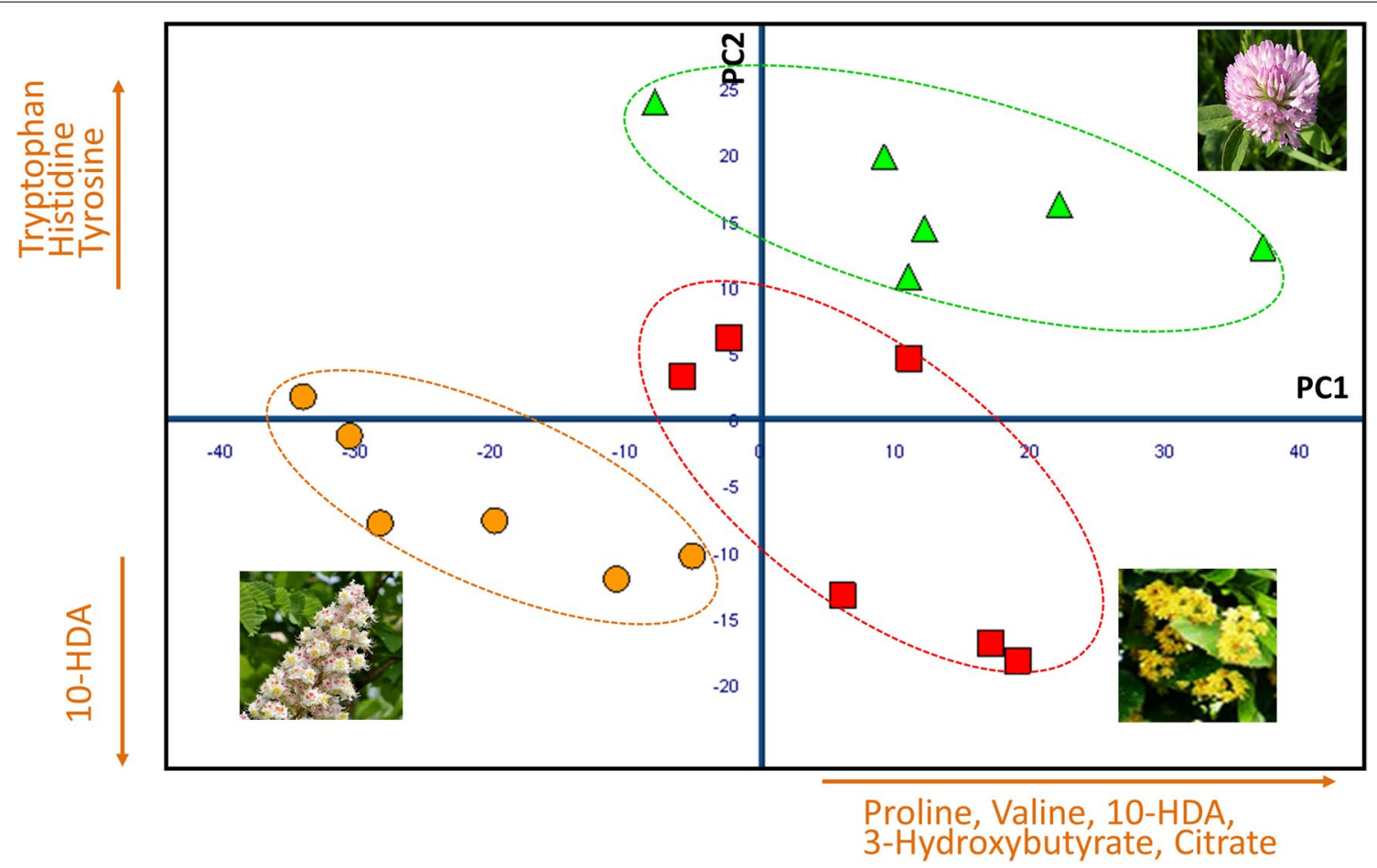

Fig. 6 PCA score-plot (58.2\% of total variance explained) based on ${ }^{1} \mathrm{H}$ NMR spectra of Royal Jelly samples produced by three Italian farms (Bo1, Bo6 and Gr5) on the 13th of June 2016 and deriving from a monofloral production conducted in the presence of either chestnut (orange circles), trifolium (green triangles) or linden (red squares) flowerings. The ellipses were arbitrarily generated to indicatively group the different RJ types. The name and orthogonal direction of most significant loading vectors involved in the differentiation among RJ types are reported along the plot borders (more details are reported in Additional file 1: Fig. S12)

to easily discriminate Italian high quality royal jellies from less valuable and controlled imported products. Although our findings should still be considered preliminary, due to the relatively small number of RJ replicates employed here, they are, however, altogether promising and encourage to adopt the proposed combined NMR and statistical approach over a larger number of samples to enlarge and further validate a spectral database of royal jelly products.

\section{Supplementary information}

Supplementary information accompanies this paper at https://doi. org/10.1186/s40538-020-00190-8.

Additional file 1: Fig. S1. Pollen traps fastened at the hive entrance and enabling the pollen stripping and collection. The pollen accumulates in a side opening drawer when the bees, during their access into the hive, walk through the metallic mesh which connects the pollen trap to the landing board of the hive. The trap is composed of round shaped holes that strip the pollen grains from either the pollen basket or the corbicula on outside of the tibia on the hind legs of the bees. Fig. S2. ${ }^{1} \mathrm{H}-\mathrm{NMR}$ spectrum of a representative Italian $\mathrm{RJ}$ dissolved in phosphate buffer solution $(0.2 \mathrm{M}, \mathrm{pH} 7.3)$ prepared with $d_{2} \mathrm{O}$ and containing TMSPA $(d=0 \mathrm{ppm})$ as internal standard. The proton acquisition was carried out by applying the presaturation technique to suppress the residual water signal at $\approx 4.704$ ppm. Fig. S3. $2 \mathrm{D}^{1} \mathrm{H}-{ }^{-} \mathrm{H}$ NMR COSY spectrum of a representative Italian RJ dissolved in phosphate buffer solution $\left(0.2 \mathrm{M}, \mathrm{pH}\right.$ 7.3) prepared with $d_{2} \mathrm{O}$ and containing TMSPA ( $d=0 \mathrm{ppm}$ ) as internal standard. The acquisition was carried out by applying the presaturation technique to suppress the residual water signal at $\approx 4.704 \mathrm{ppm}$. Fig. $\mathbf{S 4}$. Superimposition of $2 \mathrm{D}^{1} \mathrm{H}$ ${ }^{13} \mathrm{C}$ NMR HSQC (red) and HMBC (blue) spectra of a representative Italian RJ dissolved in phosphate buffer solution $\left(0.2 \mathrm{M}, \mathrm{pH}\right.$ 7.3) prepared with $d_{2} \mathrm{O}$ and containing TMSPA as internal standard. Fig. S5. Significant loading vectors related to the PCA score-plot shown in Fig. 3 and based on ${ }^{1} \mathrm{H}$ NMR spectra of Royal Jelly samples produced from two Bologna (Bo1 and Bo6) and four Grosseto (Gr2, Gr3, Gr4 and Gr5) farms on the 30th of May 2016. Fig. S6. PLS-DA results based on ${ }^{1}$ H NMR spectra of Royal Jelly samples produced from Bologna (squares) and Grosseto (circles) farms on the 30th of May 2016. (A) PLS-DA Cross-validation score-plot, where full and holed objects differentiate samples belonging to either the training or the test set, respectively. (B) PLS-DA classification results and Q2/R2 values related to the PLS-DA components 1 and 2; (C) PLS-DA cross-validation results.Fig. S7. PLS-DA results based on ${ }^{1} H$ NMR spectra of Royal Jelly samples produced from the two Bologna farms Bol (blue squares) and Bo6 (green squares) on the 30th of May 2016. (A) PLS-DA Cross-validation score-plot, where full and holed squares differentiate samples belonging to either the training or the test set, respectively. (B) PLS-DA classification results and Q2/R2 values related to the PLS-DA components 1 and 2; (C) PLS-DA cross-validation results. Fig. S8. PLS-DA results based on ${ }^{1} H$ NMR spectra of Royal Jelly samples produced from the four Grosseto farms Gr2, Gr3, Gr4 and GR5 (light green, dark green, orange and red circles, respectively) on the 30th of May 2016. (A) PLS-DA Cross-validation score-plot, where full and holed circles differentiate samples belonging to either the training or the test set, respectively. (B) PLS-DA classification. results and Q2/R2 values related to the PLS-DA components 1 and 2; (C) PLS-DA cross-validation results. Fig. S9. Significant loading vectors related to the PCA score-plot shown in Fig. 4 and based on ${ }^{1} \mathrm{H}$ NMR spectra of Royal Jelly samples of Italian (produced the 8th of July 2016) and 
Chinese (distributed in 2016) geographical origin. Fig. S10. PLS-DA results based on ${ }^{1} H$ NMR spectra of Royal Jelly samples of Italian and Chinese geographical origin. (A) PLS-DA Cross-validation score-plot, where full and holed objects differentiate samples belonging to either the training or the test set, respectively. (B) PLS-DA classification results and Q2/R2 values related to the PLS-DA component 1; (C) PLS-DA cross-validation results. Fig. S11. Significant loading vectors related to the PCA score-plots shown in Fig. 5A (A), 5B (B) and 5C (C) and based on ${ }^{1} \mathrm{H}$ NMR spectra of Royal Jelly samples produced by three Grosseto farms in different days (see the legend). The label 3-hydroxy correspond to 3-hydroxybutyrate. Fig. S12. Significant loading vectors related to the PCA score-plots shown in Fig. 6 and based on 'H NMR spectra of Royal Jelly samples produced by three Italian farms (Bo1, Bo6 and Gr5) on the 13th of June and deriving from a monofloral production conducted in the presence of either chestnut, trifolium or linden flowerings. Table S1. The table reports the name and the most relevant information related to the Italian farms involved in the project. Table S2. Results of ANOVA test (Benjamini-Hochberg's test, $a=0.05$ ) informing on the significance of the most discriminant variables associated to the PCs of score-plots shown in Figs. 3 and 4, respectively, and explaining a total variance $<20 \%$. Table S3. Results of ANOVA test (Benjamini-Hochberg's test, $a=0.05$ ) informing on the significance of the most discriminant variables associated to PCs of score-plots shown in Figs. 5A, 5B, 5C and 6, respectively, and explaining a total variance $<20 \%$.

\section{Abbreviations}

RJ: Royal jelly; NMR: Nuclear Magnetic Resonance; ANOVA: Analysis of variance: PCA: Principal Component Analysis; PLS-DA: Partial least square-discriminant analysis; COPAIT: Associazione per la Produzione di pappa reale italiana; $\mathrm{Bo}$, Gr and An: Farms in Bologna, Grosseto and Andria provinces, respectively; Ch: Chinese RJ; t, c and t: Trifolium, chestnut and linden pollen, respectively; TMSPA: 3-(Trimethylsilyl) propionic-2,2,3,3-d4 acid; COSY: COrrelation SpectroscopY; TOCSY: TOtal Correlation SpectroscopY; NOESY: Nuclear Overhauser Effect SpectroscopY; HSQC: Heteronuclear single-quantum coherence; HMBC: Heteronuclear multiple-bond coherence; 10-HDA: 10-Hydroxy-2-decenoic acid; NIC, ADE GUA and URI: Nicotinamidic-, adenosinic-, guanosinic- and uridinic-like structures, respectively.

\section{Acknowledgements}

All NMR experimental work was conducted at CERMANU, Università di Napoli Federico Il.

\section{Authors' contributions}

$\mathrm{PM}, \mathrm{AP}$ and $\mathrm{EC}$ have made substantial contributions to the conception, design and revision of the work; PM and MB have acquired, analysed and interpreted NMR data. PM have drafted the work. All authors read and approved the final manuscript.

\section{Fundings}

A supporting fund was given to CERMANU by the Italian consortium of royal jelly producers "COPAIT, l'Associazione per la produzione e valorizzazione della pappa reale fresca italiana".

This work was partially supported by the research project "Tecniche di risonanza magnetica nucleare per discriminare campioni di pappa reale in funzione dellorigine geografica, del periodo di produzione e del tipo di fioriture accessibiliFARB-Fondi di Ateneo per la Ricerca di Base 2018 - ORSA188777" funded by the University of Salerno.

\section{Availability of data and materials}

The datasets used and/or analysed during the current study are available from the corresponding author on reasonable request.

\section{Ethics approval and consent to participate}

This manuscript is an original paper and has not been published in other journals. The authors agreed to keep the copyright rule.

\section{Consent for publication}

The authors agreed to the publication of the manuscript in this journal.

\section{Competing interests}

The authors declare that they have no competing interests.

\section{Author details}

${ }^{1}$ Centro Interdipartimentale sulla Risonanza Magnetica Nucleare per I'Ambiente, I'Agro-Alimentare ed i Nuovi Materiali (CERMANU), Università di Napoli Federico II, Via Università 100, 80055 Portici, Italy. ${ }^{2}$ Dipartimento di Farmacia (DIFARMA), Università degli Studi di Salerno, 84084 Fisciano, Italy. ${ }^{3}$ Dipartimento di Agraria, Università di Napoli Federico II, Via Università 100, 80055 Portici, Italy.

Received: 24 July 2020 Accepted: 3 September 2020

Published online: 04 November 2020

\section{References}

1. Kunugi H, Ali AM. Royal jelly and its components promote healthy aging and longevity: from animal models to humans. Int J Mol Sci. 2019;20(4662):1-26.

2. Ramadan MF, Al-Ghamdi A. Bioactive compounds and health-promoting properties of royal jelly: a review. J Funct Food. 2012;4:39-52.

3. Wytrychowski M, Chenavas S, Daniele G, Casabianca H, Batteau M, Guibert S, Brion B. Physicochemical characterisation of French royal jelly: comparison with commercial royal jellies and royal jellies produced through artificial bee-feeding. J Food Compos Anal. 2013;29:126-33.

4. Takenaka T, Takenaka Y. Royal jelly from Apis cerana japonica and Apis mellifera. Biosci Biotech Bioch. 1996;60(3):518-20.

5. Garcia-amoedo LH, Almeida-Muradian LB. Physicochemical composition of pure and adulterated royal jelly. Quím Nova. 2007;30(2):257-9.

6. Viuda-Martos M, Ruiz-Navajas Y, Fernández-López J, Pérez-Álvarez JA. Functional properties of honey, propolis, and royal jelly. J Food Sci. 2008;73:117-24.

7. Pasupuleti VR, Sammugam L, Ramesh N, Gan SH. Honey, propolis, and royal jelly: a comprehensive review of their biological actions and health benefits. Oxid Med Cell Long. 2017;1259510:1-22.

8. ISO 12824-Royal jelly_Specifications. BSI Standards Publication, 2016; ISO 12824, pp. 44. https://www.sis.se/api/document/preview/920905/.

9. Mazzei P, Piccolo A. HRMAS NMR spectroscopy applications in agriculture. Chem Biol Technol Ag. 2017;4:1-11. https://doi.org/10.1186/s4053 8-017-0093-9.

10. Lindon JC, Nicholson JK, Holmes E. The handbook of metabonomics and metabolomics, in metabonomics and metabolomics techniques and their applications. In: Lindon JC, Nicholson JK, Holmes E, editors. Mammalian system. Amsterdam: Elsevier; 2007. p. 1-35.

11. Mannina L, Sobolev A, Proietti M, Capitani D, Mazzei P, Piccolo A, et al. NMR Methodologies in food analysis. In: Mannina L, Sobolev A, Proietti $M$, editors. Analytical Chemistry-Developments, Applications, and challenges in food analysis. Hauppauge: Nova Science Publishers Inc.; 2017. p. 103-56.

12. Mazzei P, Piccolo A. ${ }^{1} \mathrm{H}$-HRMAS-NMR metabolomic to assess quality and traceability of mozzarella cheese from Campania buffalo milk. Food Chem. 2012;132:1620-7.

13. Miyakawa T, Liang T, Tanokura M. NMR-based metabolomics of foods. In: Bagchi D, Bagchi M, Swaroop A, editors. Genomics, proteomics and metabolomics in nutraceuticals and functional foods. New York: Wiley; 2015. p. 365-78.

14. Cagliani LR, Scano P, Consonni R. NMR-based metabolomics: quality and authenticity of plant-based foods. In: Webb G, editor. Modern magnetic resonance. Cham: Springer; 2017. p. 1-20.

15. Consonni R, Cagliani LR, Cogliati C. NMR characterization of saccharides in italian honeys of different floral sources. J Agric food Chem. 2012;60:4526-34. https://doi.org/10.1021/jf3008713.

16. Kortesniemi M, Slupsky CM, Ollikka T, Kauko L, Spevacek AL, Sjövall O, Yang N, Kallio H. NMR profiling clarifies the characterization of Finnish honeys of different botanical origins. Food Res Int. 2016;86:83-92.

17. Kameda T, Tamada Y. Variable-temperature 13C solid-state NMR study of the molecular structure of honeybee wax and silk. Int J Biol Macromol. 2009;44:64-9. 
18. Kasote DM, Pawar MV, Bhatia RS, Nandre VS, Gundu SS, Jagtap SD, Kulkarni MV. NMR based chemical profiling and biological characterisation of Indian propolis. Fitoterapia. 2017;122:52-60.

19. Messia MC, Sobolev AP, Gómez-Caravaca AM, Lamanna R, D’Amico I, Caboni MF, Marconi E, Mannina L. HR-MAS NMR metabolic profiling, furosine and (E)-10-Hydroxy-2-decenoic acid for qualitative and geographical discrimination of royal jelly. J Apicult Res. 2013;52:141-8.

20. Kodai T, Umebayashi K, Nakatani T, Ishiyama K, Noda N. Compositions of royal jelly II. Organic acid glycosides and sterols of the royal jelly of honeybees (Apis mellifera). Chem Pharm Bull. 2007;55:1528-31.

21. Melliou E, Chinou I. Chemistry and Bioactivity of Royal Jelly from Greece. J Agr Food Chem. 2005;53:8987-92.

22. Dimou M, Goras G, Thrasyvoulou A. Pollen analysis as a means to determine the geographical origin of royal jelly. Grana. 2007:46:118-22.

23. Mauriello G, De Prisco A, Di Prisco G, La Storia A, Caprio E. Microbial characterization of bee pollen from the Vesuvius area collected by using three different traps. PLOS ONE. 2017;12(9):1-17.

24. Louveaux J, Maurizio A, Vorwohl G. International commission for bee botany of IUBS — methods of melissopalynology. Bee World. 1978;59(4):139-57.

25. Marconi E, Caboni MF, Messia MC, Panfili G. Furosine: a suitable marker for assessing the freshness of royal jelly. J Agric Food Chem. 2002;50:2825-9.

26. Messia MC, Caboni MF, Marconi E. Storage stability assessment of freeze-dried royal jelly by furosine determination. J Agric Food Chem 2005;53:4440-3.

27. Brereton RG. Pattern recognition. Chemometrics: data analysis for the laboratory and chemical plant. England: Wiley, pp. 183-249 (2003).

28. Worley B, Powers R. Multivariate Analysis in Metabolomics. Curr. Metabolomics. 2013:1:92-107.
29. Mazzei P Celano G, Palese AM, Lardo E Drosos M Piccolo A. HRMAS-NMR metabolomics of Aglianicone grapes pulp to evaluate terroir and vintage effects, and as assessed by the electromagnetic induction (EMI) technique, spatial variability of vineyard soils. Food Chem. 2019;283:215-23.

30. Blum MS, Novak AF, Taber S. 10-Hydroxy- $\triangle 2$-decenoic acid, an antibiotic found in royal jelly. Science. 1959;21:452-3.

31. Vucevic D, Melliou E, Vasilijic S, Gasic S, Ivanovski P, Chinou I, Colic M. Fatty acids isolated from royal jelly modulate dendritic cell-mediated immune response in vitro. Int Immunopharmacol. 2007;7:1211-20.

32. Izuta H, Chikaraishi Y, Shimazawa M, Mishima S, Hara H. 10-Hydroxy-2-decenoic Acid, a major fatty acid from Royal Jelly, inhibits VEGF-induced angiogenesis in human Umbilical Vein Endothelial Cells. Evid Based Compl Alternat Med. 2009;6(4):489-94.

33. Morita H, Ikeda T, Kajita K, Fujioka K, Mori I, Okada H, Uno Y. Effect of royal jelly ingestion for six months on healthy volunteers. Nutr J. 2012;11:1-7.

34. Yang YC, Chou WM, Widowati DA, Lin IP, Peng CC. 10-hydroxy-2-decenoic acid of royal jelly exhibits bactericide and anti-inflammatory activity in human colon cancer cells. BMC Compl Altern M. 2018;18(202):1-7.

35. Hair JF, Sarstedt M, Hopkins L, Kuppelwieser VG. Partial least squares structural equation modeling (PLS-SEM) an emerging tool in business research. Eur Bus Rev. 2014;26:106-21.

36. Dimou M, Tananaki C, Goras G, Kanelis D, Thrasyvoulou A. The effect of pollen supplements on the determination of the geographical origin of royal jelly. J Apicult Res. 2014;53:124-8.

\section{Publisher's Note}

Springer Nature remains neutral with regard to jurisdictional claims in published maps and institutional affiliations. 